\title{
HIFI observations of warm gas in DR21: Shock versus radiative heating ${ }^{\star}$
}

\author{
V. Ossenkopf ${ }^{1,2}$, M. Röllig ${ }^{1}$, R. Simon ${ }^{1}$, N. Schneider ${ }^{3}$, Y. Okada ${ }^{1}$, J. Stutzki ${ }^{1}$, M. Gerin ${ }^{4}$, M. Akyilmaz ${ }^{1}$, \\ D. Beintema ${ }^{2}$, A. O. Benz ${ }^{5}$, O. Berne ${ }^{6}$, F. Boulanger ${ }^{7}$, B. Bumble ${ }^{8}$, O. Coeur-Joly ${ }^{9,10}$, C. Dedes ${ }^{5}$, \\ M. C. Diez-Gonzalez ${ }^{11}$, K. France ${ }^{12}$, A. Fuente ${ }^{13}$, J. D. Gallego ${ }^{11}$, J. R. Goicoechea ${ }^{14}$, R. Güsten ${ }^{15}$, A. Harris ${ }^{16}$, \\ R. Higgins ${ }^{17,1}$, B. Jackson ${ }^{2}$, C. Jarchow ${ }^{18}$, C. Joblin ${ }^{9,10}$, T. Klein ${ }^{15}$, C. Kramer ${ }^{19}$, S. Lord ${ }^{20}$, P. Martin ${ }^{12}$, \\ J. Martin-Pintado ${ }^{14}$, B. Mookerjea ${ }^{21}$, D. A. Neufeld ${ }^{22}$, T. Phillips ${ }^{23}$, J. R. Rizzo ${ }^{14}$, F. F. S. van der Tak ${ }^{2,24}$, \\ D. Teyssier ${ }^{25}$, and H. Yorke ${ }^{8}$
}

(Affiliations are available in the online edition)

Received 30 March 2010 / Accepted 20 April 2010

\section{ABSTRACT}

Context. The molecular gas in the DR21 massive star formation region is known to be affected by the strong UV field from the central star cluster and by a fast outflow creating a bright shock. The relative contribution of both heating mechanisms is the matter of a long debate.

Aims. By better sampling the excitation ladder of various tracers we provide a quantitative distinction between the different heating mechanisms. Methods. HIFI observations of mid- $J$ transitions of $\mathrm{CO}$ and $\mathrm{HCO}^{+}$isotopes allow us to bridge the gap in excitation energies between observations from the ground, characterizing the cooler gas, and existing ISO LWS spectra, constraining the properties of the hot gas. Comparing the detailed line profiles allows to identify the physical structure of the different components.

Results. In spite of the known shock-excitation of $\mathrm{H}_{2}$ and the clearly visible strong outflow, we find that the emission of all lines up to $\gtrsim 2 \mathrm{THz}$ can be explained by purely radiative heating of the material. However, the new Herschel/HIFI observations reveal two types of excitation conditions. We find hot and dense clumps close to the central cluster, probably dynamically affected by the outflow, and a more widespread distribution of cooler, but nevertheless dense, molecular clumps.

Key words. ISM: structure - ISM: kinematics and dynamics - ISM: molecules - HII regions - submillimeter: ISM

\section{Introduction}

DR21 is a deeply embedded H II region created by the radiation from at least six OB stars (Roelfsema et al. 1989). It sits within a ridge of dense molecular material that obscures the H II-region at optical wavelengths. The embedded cluster drives a violent bipolar outflow in north-east to south-west direction. It is prominent in the $2 \mu \mathrm{m}$ emission of vibrationally excited $\mathrm{H}_{2}$, tracing hot, shocked gas and in the Spitzer $4.5 \mu \mathrm{m}$ channel (Garden et al. 1986; Davis et al. 2007). As the cluster is located close to the eastern edge of the molecular ridge, the eastern, blue-shifted outflow expands in a blister-like fountain, while the western, redshifted outflow is highly collimated (Lane et al. 1990).

Spitzer $8 \mu \mathrm{m}$ images reveal spots of bright PAH emission with a size below 10" (Marston et al. 2004). They represent the surfaces of high-density, UV irradiated clumps, forming photon-dominated (or photo-dissociation) regions (PDRs), transition zones from ionized and atomic gas to dense molecular gas where physics and chemistry are dominated by UV radiation from young stars (Hollenbach \& Tielens 1999; Ossenkopf et al. 2007). These hot and dense regions give rise to the emission of PDR tracers, such as $\mathrm{HCO}^{+}$, high- $J \mathrm{CO}$, atomic and ionized carbon, and atomic oxygen.

An overview on the numerous existing observations in DR21 is given by Schneider et al. (2006) and Jakob et al. (2007). In

* Herschel is an ESA space observatory with science instruments provided by European-led Principal Investigator consortia and with important participation from NASA. spite of the wealth of data, the heating of the molecular gas is still debated. The bright $\mathrm{H}_{2}$ emission indicates shock heating in the outflow and the wings of the molecular lines prove its dynamical impact, but excitation models of the observed emission of CO, [CI], [C II], and [O I] by Lane et al. (1990) and Jakob et al. (2007) have shown that the emission of those tracers cannot be explained by shocks, but is consistent with a pure UV heating, i.e., PDR physics.

To quantify the heating of the gas, we analyze spectra taken with the HIFI instrument (de Graauw et al. 2010) on board the Herschel Space Observatory (Pilbratt et al. 2010) during the performance verification campaign. Observations of high- $J$ $\mathrm{HCO}^{+}$transitions trace hot material, ionized by UV radiation or X-rays (Sternberg \& Dalgarno 1995). Hot water lines are produced in shocked gas (Snell et al. 2005), and observations of CO isotopes around $J=10$ close the gap in the excitation ladder between ground-based observations and existing ISO data, allowing to obtain a full picture of the temperature distribution.

In Sect. 2 we present the observational data. Section 3 compares the measured line profiles to distinguish different components based on their velocity distribution. In Sect. 4 we provide a model for the emission, supporting the PDR character of the source, and discuss the results in Sect. 5.

\section{Observations}

\subsection{HIFI observations}

All spectra presented here were obtained in performance verification observations for the HIFI instrument. As their main goal 
Table 1. Summary of the used HIFI observational data.

\begin{tabular}{|c|c|c|c|c|}
\hline Transition & $\begin{array}{r}v_{\text {line }} \\
{[\mathrm{GHz}]}\end{array}$ & $\begin{array}{l}H P B W \\
\quad \text { " Observing mode }\end{array}$ & $\begin{array}{r}t_{\text {int,source }} \\
{[\mathrm{s}]}\end{array}$ & $\begin{array}{l}\mathrm{rms} \\
{[\mathrm{K}]}\end{array}$ \\
\hline $\mathrm{HCO}^{+} 6-5$ & 535.062 & 40 OTF map & 16 & 0.04 \\
\hline $\mathrm{HCO}^{+} 12-11$ & 1069.694 & 21 FSW spectral scan & 150 & 0.1 \\
\hline${ }^{13} \mathrm{CO} 10-9$ & 1101.350 & 21 LC spectral scan & 270 & 0.08 \\
\hline $\mathrm{C}^{18} \mathrm{O} 10-9$ & 1097.163 & 21 LC spectral scan & 270 & 0.08 \\
\hline \multirow[t]{3}{*}{ [C II] } & 1900.537 & 12 DBS raster map & 14 & 1 \\
\hline & & 20 convolved+binned & 112 & 0.2 \\
\hline & & 40 convolved+binned & 420 & 0.1 \\
\hline
\end{tabular}

Notes. ${ }^{(1)}$ DBS = dual-beam-switch, OTF = On-The-Fly, FSW $=$ frequency-switch, LC $=$ load-chop, OFF position $=20 \mathrm{~h} 37 \mathrm{~m} 10 \mathrm{~s}$, $42^{\circ} 37^{\prime} 00^{\prime \prime}$

was to demonstrate the functionality and performance of the different observing modes, the spectra were taken with a large variety of observing modes and strategies. Consequently, every spectrum was taken in a slightly different manner. All observational parameters are summarized in Table 1.

Most observations where single-point observations towards the central position of the DR21 H II region at $\mathrm{RA}=20 \mathrm{~h} 39 \mathrm{~m} 01.1 \mathrm{~s}$, Dec $=42^{\circ} 19^{\prime} 43.0^{\prime \prime}(\mathrm{J} 2000)$. Fully sampled maps were only obtained in the [CII] line. Data were taken with the wideband spectrometer (WBS) at a resolution of $1.1 \mathrm{MHz}$, corresponding to $0.2 \mathrm{~km} \mathrm{~s}^{-1}$ (at $1900 \mathrm{GHz}$ ) $0.7 \mathrm{~km} \mathrm{~s}^{-1}$ (at $500 \mathrm{GHz}$ ). The [CII] data were rebinned to a velocity resolution of $0.45 \mathrm{~km} \mathrm{~s}^{-1}$ to improve the signal to noise.

\subsection{Complementary data}

ISO long wavelength spectrometer 43-197 $\mu \mathrm{m}$ grating scans were obtained for the DR21 central position from the ISO Data Archive (TDT 15200786). Integrated line intensities were extracted for [O I] at 63 and $145 \mu \mathrm{m}$ and the CO 14-13 to 17-16 transitions. Mid- $J$ CO lines of the DR21 region were mapped with the KOSMA 3 m submm telescope (Jakob et al. 2007). We use the lines of $\mathrm{CO}$ and ${ }^{13} \mathrm{CO}$ from $J=3-2$ to $7-6$, which have been observed at native angular resolutions from $80^{\prime \prime}$ to $40^{\prime \prime}$. The $\mathrm{HCO}^{+} 1-0, \mathrm{H}^{13} \mathrm{CO}^{+} 1-0$, and $\mathrm{HCO}^{+} 3-2$ observations were taken with the IRAM 30 m telescope (Schneider et al. 2010). Native angular resolutions at the 1-0 and 3-2 transition frequencies are $28^{\prime \prime}$ and $9^{\prime \prime}$, respectively.

\subsection{Beam size effects}

For a direct comparison of the different data sets we smoothed the available data to the coarsest common angular resolution of $80^{\prime \prime}$, matching that of the CO 3-2 KOSMA beam and the lowest frequency ISO observations. This is impossible for the single-point HIFI observations. Moreover, the Herschel beam varies between $40^{\prime \prime} \mathrm{HPBW}$ for the $\mathrm{HCO}^{+}$line at $535 \mathrm{GHz}$ and $20^{\prime \prime}$ for ${ }^{13} \mathrm{CO}$ at $1100 \mathrm{GHz}$. Those data were corrected for the different beam filling by estimating the source size from the $\mathrm{HCO}^{+}$3-2 line as a PDR tracer (Sternberg \& Dalgarno $1995)$ with quite compact emission (20" to $30^{\prime \prime}$ in diameter). Successively convolving from the native angular resolution of $9^{\prime \prime}$ to the angular resolution of the Herschel data, and further to the final, smoothed spectra at $80^{\prime \prime}$, we obtained scaling factors for the HIFI spectra, being 0.5 when going from $40^{\prime \prime}$ to $80^{\prime \prime}$ and 0.33 when going from $20^{\prime \prime}$ to $80^{\prime \prime}$. The maps obtained in [CII] allow a direct smoothing to a resolution of $40^{\prime \prime}$ (see Table 1). Beyond that size, the same scaling factor as above was applied.

\section{Line profiles}

The molecular ridge including the DR21 H II region has an intrinsic LSR velocity of $-3 \mathrm{~km} \mathrm{~s}^{-1}$. The quiescent material is Page 2 of 5

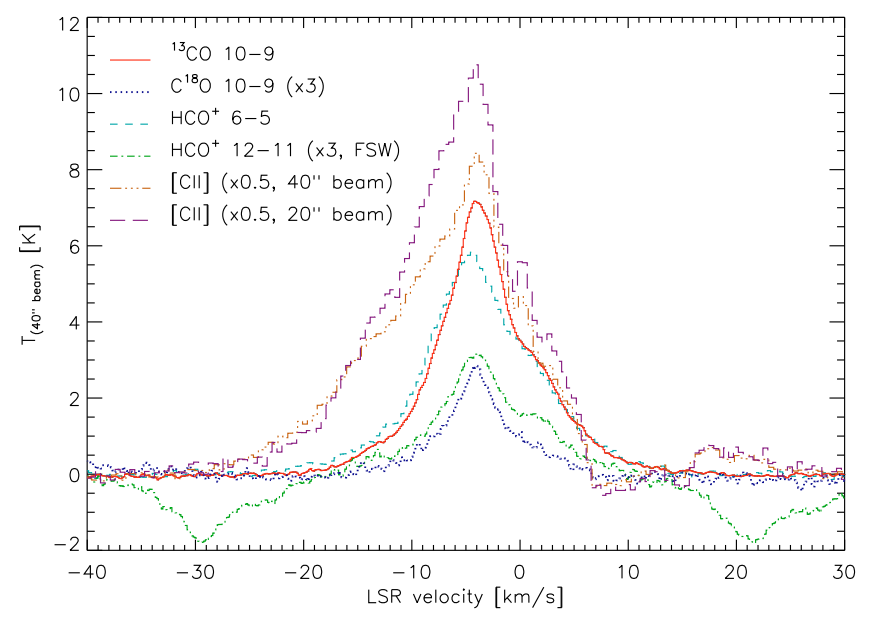

Fig. 1. HIFI spectra of the lines used in the fit of the PDR properties. The $[\mathrm{CII}]$ data are convolved either to the resolution of the $\mathrm{HCO}^{+} 6-5$ line or to that of the $\mathrm{CO} 10-9$ lines. The negative features in the $\mathrm{HCO}^{+} 12-11$ line are artifacts from the frequency-switch observing mode.

visible in narrow absorption lines of $\mathrm{NH}_{3}$ (Matsakis et al. 1977) and $\mathrm{H}_{2} \mathrm{CO}$ (Bieging et al. 1982). A second velocity component at $8-10 \mathrm{~km} \mathrm{~s}^{-1}$ is known to be associated with the $\mathrm{W} 75 \mathrm{~N}$ complex. It appears in emission in CO and [C I] (Jakob et al. 2007), as a narrow absorption feature in $\mathrm{HCO}^{+} 1-0$ (Nyman 1983), and has a very broad velocity distribution in the HI $21 \mathrm{~cm}$ absorption (Thompson et al. 1969; Roberts et al. 1997). The wings of the low- $J$ CO lines trace outflow velocities down to about $-20 \mathrm{~km} \mathrm{~s}^{-1}$ for the eastern, blister outflow and up to $\approx 10 \mathrm{~km} \mathrm{~s}^{-1}$ for the western outflow.

Figure 1 shows the profiles of the HIFI spectra of CO isotopes, $\mathrm{HCO}^{+}$and $[\mathrm{C} \mathrm{II}]$. All lines peak at about $-4 \mathrm{~km} \mathrm{~s}^{-1}$. The $\mathrm{CO}$ and $\mathrm{HCO}^{+}$lines have a similar shape, but the $[\mathrm{C}$ II] line shows an additional broad blue wing extending down to $-30 \mathrm{~km} \mathrm{~s}^{-1}$. This indicates that the warm molecular material is slightly blue-shifted relative to the cold gas and that the [C II] emission is not only originating from that warm gas, but also from the ionized wind in the blister outflow.

Figure 2 compares the shapes of the $\mathrm{CO}$ and $\mathrm{HCO}^{+}$isotope lines with complementary ground-based measurements towards the same positions. We show only a few selected transitions as, e.g., the data for the $\mathrm{CO} 7-6$ or 4-3 lines provide no additional information. All CO isotopic lines up to 7-6 are roughly symmetric, centered at the ridge velocity of $-3 \mathrm{~km} \mathrm{~s}^{-1}$. The lines of the main isotope are heavily self-absorbed with the absorption dip marking line center. The 10-9 lines, tracing hotter material, are slightly asymmetric and shifted to $-4 \mathrm{~km} \mathrm{~s}^{-1}$. All $\mathrm{HCO}^{+}$lines, except $\mathrm{H}^{13} \mathrm{CO}^{+} 1-0$, have a profile very similar to the 10-9 lines of the $\mathrm{CO}$ isotopes indicating that $\mathrm{HCO}^{+}$is mainly abundant in the heated layer and less in the overall molecular material. The $\mathrm{H}^{13} \mathrm{CO}^{+} 1-0$ line has a deviating profile centered at $-2 \mathrm{~km} \mathrm{~s}^{-1}$ tracing global infall (Kirby 2009). CO 3-2 and $\mathrm{HCO}^{+} 1-0$ show an additional feature at the $\mathrm{W} 75 \mathrm{~N}$ complex velocity.

To better understand the exact velocity distribution in the source, we plot some additional HIFI lines towards the same position (see Falgarone et al. 2010; Ossenkopf et al. 2010; van der Tak et al. 2010) in Fig. 3. The $1097 \mathrm{GHz}$ line of hot water matches the rest of the PDR tracers. The foreground at $-3 \mathrm{~km} \mathrm{~s}^{-1}$ is visible in absorption in the ground state transitions of water, but in emission in a nearby OFF position for [C II]. At velocities of about $+2 \mathrm{~km} \mathrm{~s}^{-1}$, we find the warm gas facing the redshifted western outflow, apparent as a secondary peak in the ground state 


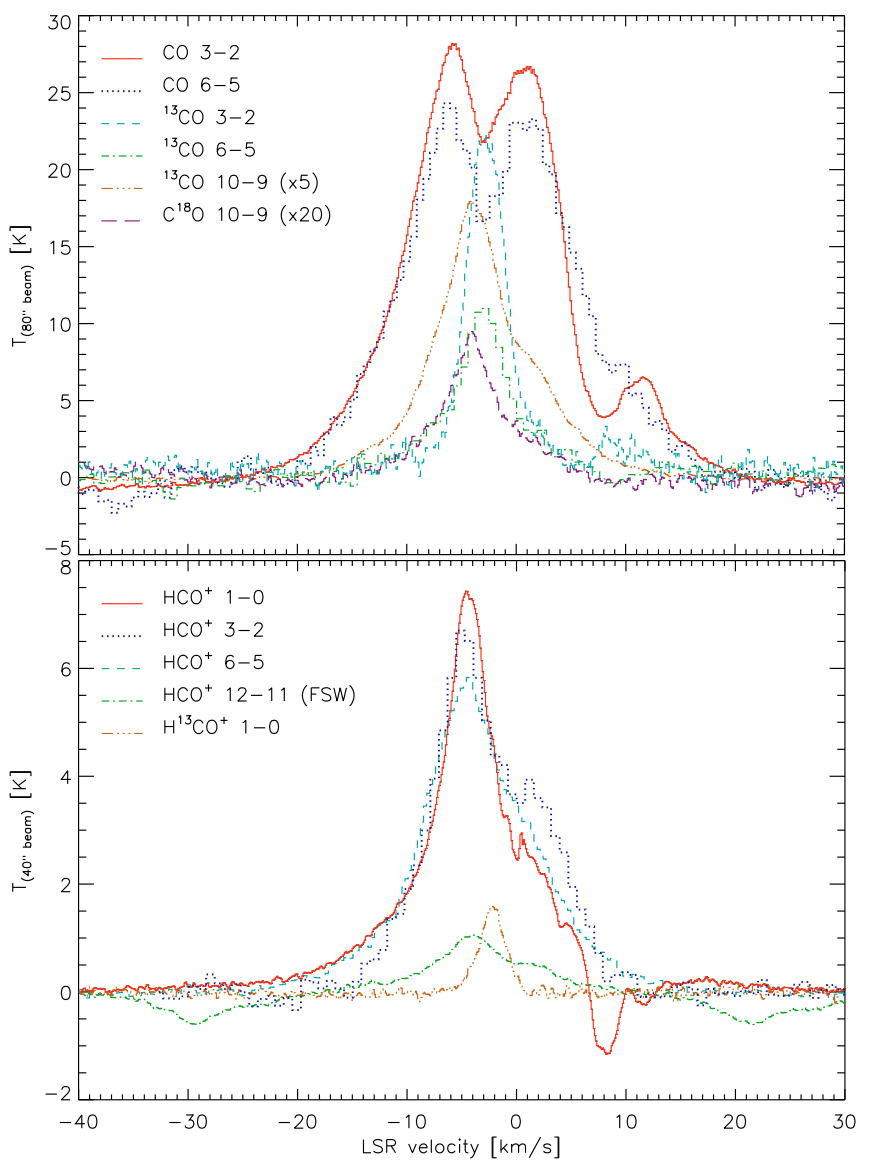

Fig. 2. Selected profiles of CO isotope lines (upper plot) and $\mathrm{HCO}^{+}$isotope lines towards the DR21 central position. All lines are convolved or scaled to the coarsest common resolution (see Sect. 2.3).

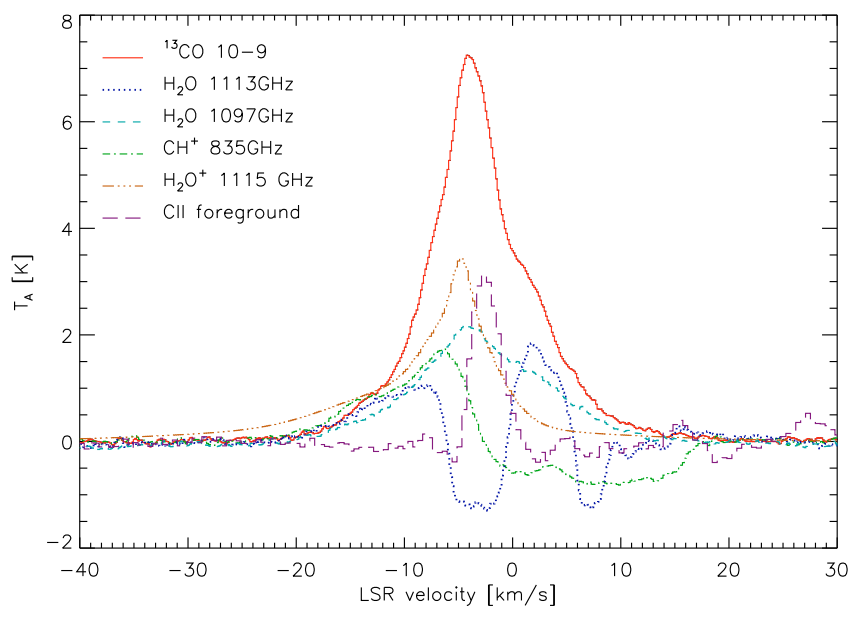

Fig. 3. Auxiliary lines measured by HIFI towards the same position. They can be used to estimate the contribution of the foreground material. The ${ }^{13} \mathrm{CO} 10-9$ profile is also displayed for comparison.

water and $\mathrm{CH}^{+}$lines and as a shoulder in ${ }^{13} \mathrm{CO}$ and the hot water line. Finally, we can clearly identify the cold W75N component at $\approx-9 \mathrm{~km} \mathrm{~s}^{-1}$.

For the warm gas we can distinguish two velocity components - a blue shifted one related to the blister outflow at $-4 \mathrm{~km} \mathrm{~s}^{-1}$ and a second one, at $2 \mathrm{~km} \mathrm{~s}^{-1}$, related to the western outflow. This is consistent with the clumpy PDR geometry proposed by Lane et al. (1990). In terms of line modelling, it is, however, impossible to separate the two outflow directions as their emission is overlapping to a large degree. We simply add their intensities. The $[\mathrm{C}$ II] emission requires a special treatment because of the additional emission from the ionized outflow. To take this into account, we have obtained an integrated [C II] intensity by scaling the ${ }^{13} \mathrm{CO} 10-9$ line profile to match the peak and the red wing of the $[\mathrm{C} \mathrm{II}]$ profile and ignoring the remaining blue-wing emission.

\section{Modelling}

We use the KOSMA- $\tau$ PDR code (Röllig et al. 2006) to model the emission of PDR ensembles, representing a distribution of spherical clumps with $\mathrm{d} N / \mathrm{d} M \propto M^{-1.8}$ (Cubick et al. 2008). For DR21 two ensembles with different properties had to be superimposed, a hot component, close to the inner H II region with strong FUV illumination, but only a small fraction of the total mass, and a cooler component that fills a larger solid angle and provides the bulk of the material. Each clumpy PDR ensemble has five free parameters: the average ensemble density, $n_{\mathrm{ens}}$, the ensemble mass, $M_{\mathrm{ens}}$, the UV field strength, $\chi$ given in units of the Draine field, and the minimum and maximum mass of the clump ensemble, $\left[M_{\min }, M_{\max }\right]$. In contrast to most other PDR models, we fit absolute line intensities, using the available ground-based observations, complementary ISO data, and the $\mathrm{HIFI}$ lines of the $\mathrm{CO}$ isotopes, $\mathrm{HCO}^{+}$, atomic and ionized carbon, and atomic oxygen. The chemical network that has been applied in these calculations includes ${ }^{13} \mathrm{C}$ but not ${ }^{18} \mathrm{O}$. The $\mathrm{C}^{18} \mathrm{O}$ lines were scaled from the ${ }^{13} \mathrm{CO}$ intensities with a conversion of 1:8. Simulated annealing was used to find the optimum parameter combination.

The significance of the model is limited by the fact that the clump superposition ignores mutual line shading between different clumps, i.e., optical depth effects are only considered within individual clumps. This is usually justified by the virialised velocity dispersion between different clumps, but for optically very thick and broad lines some correction is needed. To estimate the effect we have computed the optical depth for the bulk of the individual clumps. This is of the order of unity or below for the majority of the observed transitions, reaches values up to ten for ${ }^{13} \mathrm{CO}$ and $\mathrm{HCO}^{+}$transitions up to $J=4$, but exceeds ten for the CO main isotope lines up to $J=6$ and the [O I] $63 \mu \mathrm{m}$ line. For the $\mathrm{CO}$ lines showing clear self-absorption dips, we performed a Gaussian fit to the line wings and used the integrated intensity of that Gaussian to compute the total emission including the blocked radiation from the inner clumps close to the H II-region. As we have no spectral information for the [OI] line, we have no estimate for the blocked radiation in this case, so that we excluded that data point from the fit.

The best fit result is shown in Fig. 4. The corresponding model parameters are:

$$
\begin{aligned}
\text { Ensemble 1: } & \chi=1.0 \times 10^{5}, n_{\mathrm{ens}}=1.3 \times 10^{6} \mathrm{~cm}^{-3} \\
& M_{\mathrm{ens}}=150 M_{\odot},\left[M_{\min }, M_{\mathrm{max}}\right]=\left[10^{-2}, 8 \times 10^{1}\right] M_{\odot} \\
\text { Ensemble 2: } & \chi=3.2 \times 10^{2}, n_{\mathrm{ens}}=1.1 \times 10^{6} \mathrm{~cm}^{-3}, \\
& M_{\mathrm{ens}}=830 M_{\odot},\left[M_{\min }, M_{\max }\right]=\left[10^{-3}, 10^{1}\right] M_{\odot} .
\end{aligned}
$$

The two-ensemble model provides a reasonable fit to all 22 data points. The model slightly overestimates the emission for the optically thick rotational lines of ${ }^{13} \mathrm{CO}$ and $\mathrm{HCO}^{+}$which showed no clear self-absorption dip, so that we have insufficient optical depth corrections. The reduced $\chi^{2}$ value of the fit amounts to 11.1 , where a contribution of 9.5 results from the three optically thick low- $J$ data points of ${ }^{13} \mathrm{CO}$ and $\mathrm{HCO}^{+}$. For the remaining 19 data points we would obtain an excellent reduced $\chi^{2}$ value of 1.6. The topology of the $\chi^{2}$ function shows several side minima, but they are worse by at least a factor two. As expected, the 

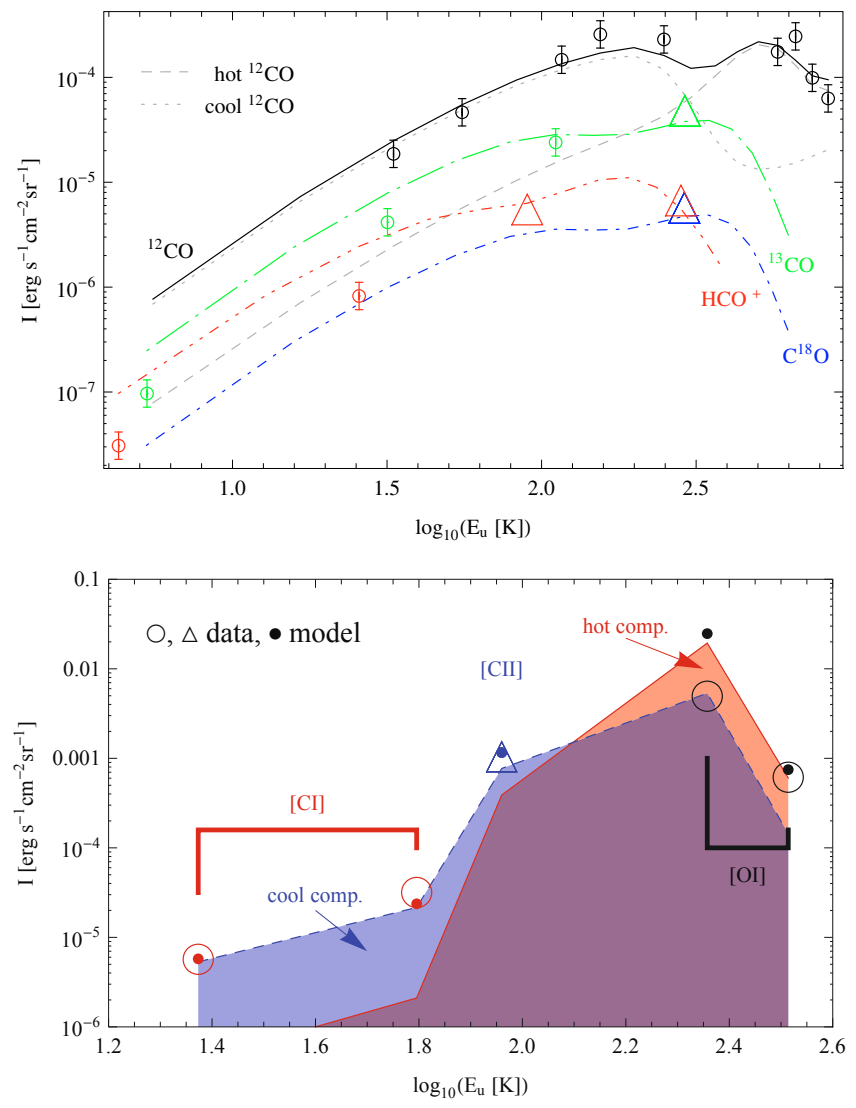

Fig. 4. Two-ensemble PDR model fit to the observed $\mathrm{CO}, \mathrm{HCO}^{+}$, and fine structure line intensities, shown as function of the upper level energy. HIFI measurements are depicted as open triangles, complementary data points as open circles. The dotted line and the shaded areas indicate the contributions from the two ensembles to the $\mathrm{CO}$ and the fine structure lines, respectively.

model predicts a too high [OI] intensity, as it ignores that the outer clumps of the cooler ensemble block the contribution from the hot inner component.

\section{Discussion}

The double-peak structure of the predicted CO intensities across the excitation ladder reflects the two different UV fields leading to different excitation conditions. No single-parameter ensemble can fit all data, but distinct low temperature and high temperature components are needed. The parameters of the model are in agreement with independent estimates. A UV flux of $10^{5}$ Draine fields corresponds to a geometrical distance of $0.06 \mathrm{pc}$, i.e. $7^{\prime \prime}$, from the central cluster, matching the size of the PAH emission (Marston et al. 2004). Our clump densities match those determined by Jakob et al. (2007) for the extended cool gas, but are slightly higher than their hot-gas density $\left(4 \times 10^{5} \mathrm{~cm}^{-3}\right)$. In contrast, Jones et al. (1994); van der Tak et al. (2010) find still somewhat higher densities for the hot gas, up to $10^{7} \mathrm{~cm}^{-3}$. The hot ensemble mass is close to the $170 M_{\odot}$ derived from early CO 7-6 observations by Jaffe et al. (1989). The total mass of the PDR ensembles falls between the mass limits derived by Jakob et al. (2007) from dust observations and from line radiative transfer fits.

While the existing ground-based observations provide a very good constraint on the properties of the extended cool gas, and the ISO lines show the total amount of hot gas, it is only the set of new HIFI data that puts the hot and cold distributions well apart from each other in terms of the temperature structure. While Jakob et al. (2007) obtained cooling curves with single peaks, the new data for the 10-9 lines of the CO isotopes and the $\mathrm{HCO}^{+}$transitions force the fit to a bimodal distribution of excitation conditions. When we exclude the Herschel data from the model fit, we obtain a parameter set that shows a UV field that is lower by a factor ten for the hot ensemble, i.e., that would imply molecular clumps farther away from the central cluster. For the cold ensemble, the fitted UV field is also somewhat lower, while all other parameters remain similar to those from the full fit. Only with the Herschel data, we therefore obtain a parameter set that is consistent with the source geometry.

As the two-ensemble PDR model is able to fit all of the observed lines, we find no evidence for a shock heating of the dense gas. This is in agreement with the analysis of Lane et al. (1990), explicitely excluding a shock origin of the fine-structure lines, but seems to be in contradiction with the analysis of the line profiles in Sect. 3 that shows excited outflow material. We conclude that the material visible in the blue line wing, characterizing the blister outflow, is contained in dense clumps that are accelerated by the outflow, but that are chemically and energetically fully dominated by the UV field and not by the associated shock.

Acknowledgements. HIFI has been designed and built by a consortium of institutes and university departments from across Europe, Canada and the United States under the leadership of SRON Netherlands Institute for Space Research, Groningen, The Netherlands and with major contributions from Germany, France and the US. Consortium members are: Canada: CSA, U.Waterloo; France: CESR, LAB, LERMA, IRAM; Germany: KOSMA, MPIfR, MPS; Ireland: NUI Maynooth; Italy: ASI, IFSI-INAF, Osservatorio Astrofisico di Arcetri- INAF; Netherlands: SRON, TUD; Poland: CAMK, CBK; Spain: Observatorio Astronmico Nacional (IGN), Centro de Astrobiología (CSIC-INTA). Sweden: Chalmers University of Technology - MC2, RSS \& GARD; Onsala Space Observatory; Swedish National Space Board, Stockholm University - Stockholm Observatory; Switzerland: ETH Zurich, FHNW; USA: Caltech, JPL, NHSC.

This work was supported by the German Deutsche Forschungsgemeinschaft, $D F G$ project number Os 177/1-1. A portion of this research was performed at the Jet Propulsion Laboratory, California Institute of Technology, under contract with the National Aeronautics and Space Administration.

\section{References}

Bieging, J. H., Wilson, T. L., \& Downes, D. 1982, A\&AS, 49, 607 Cubick, M., Stutzki, J., Ossenkopf, V., et al. 2008, A\&A, 488, 623 Davis, C. J., Kumar, M. S. N., Sandell, G., et al. 2007, MNRAS, 374, 29 Falgarone, E., Ossenkopf, V., Gerin, M., et al. 2010, A\&A, 518, L118 Garden, R., Geballe, T. R., Gatley, I., \& Nadeau, D. 1986, MNRAS, 220, 203 de Graauw, Th., et al. 2010, 518, L6

Jaffe, D. T., Genzel, R., Harris, A. I., et al. 1989, ApJ, 344, 265

Hollenbach, D. J., \& Tielens, A. G. G. M. 1999, Rev. Mod. Phys., 71, 173

Jakob, H., Kramer, C., Simon, R., et al. 2007, A\&A, 461, 999

Jones, K. N., Field, D., Gray, M. D., \& Walker, R. N. F. 1994, A\&A, 288, 581

Kirby, L. 2009, ApJ, 694, 1056

Lane, A. P., Haas, M. R., Hollenbach, D. J., \& Erickson, E. F. 1990, ApJ, 361,132

Marston, A. P., Reach, W. T., Noriega-Crespo, A., et al. 2004, ApJS, 154, 333

Matsakis, D. N., Brandshaft, D., Chui, M. F., et al. 1977, ApJ, 214, L67

Ossenkopf, V., Röllig, M., Cubick, M., \& Stutzki, J. 2007, in Molecules in Space and Laboratory, ed. J. L. Lemaire, \& F. Combes, S. Diana publ., 95 Ossenkopf, V., Müller, H. S. P., Lis, D., et al. 2010, A\&A, 518, L111 Nyman, L.-A. 1983, A\&A, 120, 307

Pilbratt, G. L., et al. 2010, 518, L1

Roberts, D. A., Dickel, H. R., \& Goss, W. M. 1997, ApJ, 476, 209

Roelfsema, P. R., Goss, W. M., \& Geballe, T. R. 1989, A\&A, 222, 247

Röllig, M., Ossenkopf, V., Jeyakumar, S., et al. 2006, A\&A, 451, 917

Schneider, N., Bontemps, S., Simon, R., et al. 2006, A\&A, 458, 855

Schneider, N., Csengeri, T., \& Bontemps, S. 2010, A\&A, accepted [arXiv: 1003.4198]

Snell, R. L., Hollenbach, D., Howe, J. E., et al. 2005, ApJ, 620, 758

Sternberg, A., \& Dalgarno, A. 1995, ApJS, 99, 565

Thompson, A. R., Colvin, R. S., \& Hughes, M. P. 1969, ApJ, 158, 939

van der Tak, F. F. S., Marseille, M. G., Herpin, F., et al. 2010, A\&A, 518, L107 
1 I. Physikalisches Institut der Universität zu Köln, Zülpicher Straße 77, 50937 Köln, Germany

2 SRON Netherlands Institute for Space Research, PO Box 800, 9700 AV Groningen, The Netherlands

3 Laboratoire AIM, CEA/DSM - INSU/CNRS - Université Paris Diderot, IRFU/SAp CEA-Saclay, 91191 Gif-sur-Yvette, France

${ }^{4}$ LERMA \& UMR 8112 du CNRS, Observatoire de Paris and École Normale Supérieure, 24 Rue Lhomond, 75231 Paris Cedex 05, France

5 Institute for Astronomy, ETH Zürich, 8093 Zürich, Switzerland

${ }^{6}$ Leiden Observatory, Universiteit Leiden, PO Box 9513, 2300 RA Leiden, The Netherlands

7 Institut d'Astrophysique Spatiale, Université Paris-Sud, Bât. 121, 91405 Orsay Cedex, France

8 Jet Propulsion Laboratory, 4800 Oak Grove Drive, MC 302-231, Pasadena, CA 91109, USA

9 Université de Toulouse, UPS, CESR, 9 avenue du colonel Roche, 31028 Toulouse Cedex 4, France

10 CNRS, UMR 5187, 31028 Toulouse, France

11 Observatorio Astronómico Nacional (IGN), Centro Astronómico de Yebes, Apartado 148, 19080 Guadalajara, Spain

12 Department of Astronomy and Astrophysics, University of Toronto, 60 St. George Street, Toronto, ON M5S 3H8, Canada
13 Observatorio Astronómico Nacional (OAN), Apdo. 112, 28803 Alcalá de Henares (Madrid), Spain

14 Centro de Astrobiología, CSIC-INTA, 28850 Madrid, Spain

15 Max-Planck-Institut für Radioastronomie, Auf dem Hügel 69, 53121 Bonn, Germany

16 Astronomy Department, University of Maryland, College Park, MD 20742, USA

17 Experimental Physics Dept., National University of Ireland: Maynooth, Co. Kildare, Ireland

18 MPI für Sonnensystemforschung, 37191 Katlenburg-Lindau, Germany

19 Instituto de Radio Astronomía Milimétrica (IRAM), Avenida Divina Pastora 7, Local 20, 18012 Granada, Spain

20 IPAC/Caltech, MS 100-22, Pasadena, CA 91125, USA

21 Tata Institute of Fundamental Research (TIFR), Homi Bhabha Road, Mumbai 400005, India

22 Department of Physics and Astronomy, Johns Hopkins University, 3400 North Charles Street, Baltimore, MD 21218, USA

${ }^{23}$ California Institute of Technology, 320-47, Pasadena, CA 911254700, USA

24 Kapteyn Astronomical Institute, University of Groningen, PO Box 800, 9700 AV Groningen, The Netherlands

25 European Space Astronomy Centre, Urb. Villafranca del Castillo, PO Box 50727, Madrid 28080, Spain 